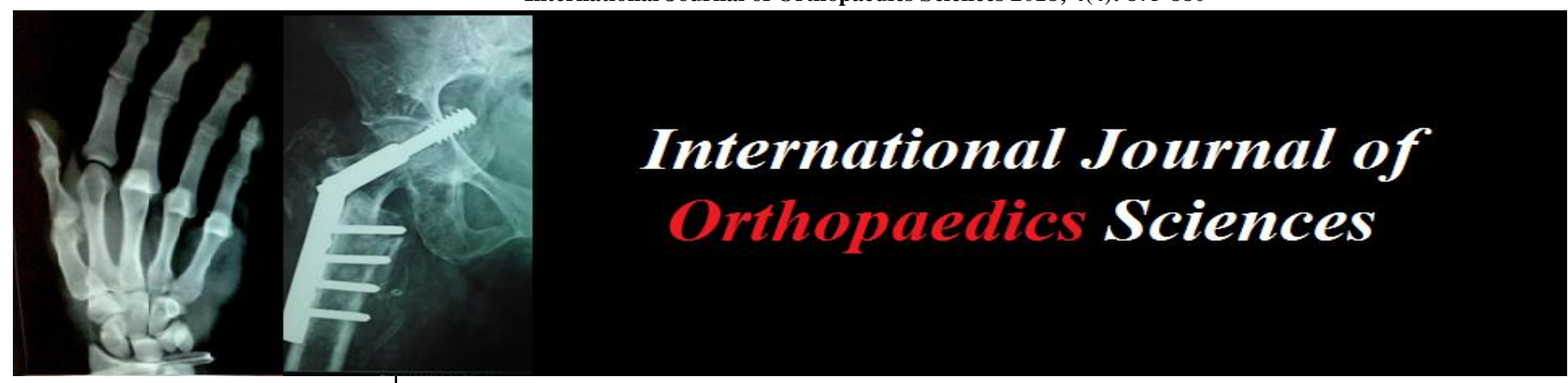

ISSN: $2395-1958$

IJOS 2018; 4(4): 875-880

(C) 2018 IJOS

www.orthopaper.com

Received: 05-08-2018

Accepted: 10-09-2018

Dr. Dinesh Loganathan

Department of Orthopaedics, Chettinad Hospital and Research Institute, Chennai, Tamil Nadu, India

\section{Dr. Soma Sundar S}

Department of Orthopaedics, Chettinad Hospital and Research Institute, Chennai, Tamil Nadu, India

Dr Shyam Sundar Ortho-One Orthopaedic Speciality Centre, Coimbatore, Tamil Nadu, India

Dr Santhosh Sahanand Ortho-One Orthopaedic Speciality Centre, Coimbatore, Tamil Nadu, India

Dr. David V Rajan Ortho-One Orthopaedic Speciality Centre, Coimbatore, Tamil Nadu, India

\section{Correspondence}

Dr. Dinesh Loganathan

Department of Orthopaedics,

Chettinad Hospital and Research Institute, Chennai, Tamil Nadu,

India

\section{Mucoid degenerated anterior cruciate ligament (ACL) managed by arthroscopic ACL reconstruction: A case series}

\author{
Dr. Dinesh Loganathan, Dr. Soma Sundar S, Dr. Shyam Sundar, Dr. \\ Santhosh Sahanand and Dr. David V Rajan
}

DOI: https://doi.org/10.22271/ortho.2018.v4.i4l.113

\section{Abstract}

Introduction: Mucoid degeneration of the Anterior Cruciate Ligament is a rare degenerative condition affecting the middle age population (16 to 50 years). The infiltration of glycosaminoglycan into the substance of the ACL causes the ligament to swell up and impinge on the intercondylar notch and Posterior Cruciate Ligament. This results in posterior knee pain and restriction of terminal movements. The current trend of management is debulking the ligament by stripping the degenerated part and preserving the native thin ACL. This poses two problems - 1. Difficulty in identifying the degenerated parts by the arthroscope. Stripping is done with the aid of pre-op MRI; 2. Chances of under stripping (recurrence of the symptoms) or over stripping (laxity and ACL failure). Studies have shown high incidence of grade 1/grade 2 laxity by Lachman test 6 months post-op and a requirement of Secondary ACL reconstruction in many patients.

Goal: The goal of this study is to assess the functional outcome of Primary ACL reconstruction with autologous quadrupled hamstring tendon graft after complete excision of the native degenerated ACL.

Materials and Methods: This is a prospective study of 13 patient ( 7 male, 6 female) of average age 41 (25-63) years presenting with posterior knee pain and restriction of flexion for an average period of 6 months (15 days - 36 months) diagnosed with Mucoid degeneration of ACL by MRI, arthroscopy and by histo-pathological examination. They were operated by ACL reconstruction following complete excision of degenerated ACL, as a single stage procedure.

Evaluation: The patients were evaluated both pre-operatively and post-operatively by Lysholm knee scoring system and VAS (visual analog score) pain scoring system.

Results: Postoperatively, patients were followed up for a minimum of 1 year (average of 14.9 months). There was no evidence of recurrence or instability at the end of follow-up. There was significant improvement in Lysholm score (34.6 to 83.2) and VAS (5.76 to 0.54).

Conclusion: Treatment of ACL mucoid degeneration by this modality is effective in preventing recurrence, post-operative laxity and provides better functional outcome compared to the classical partial resection of the degenerated ACL.

Keywords: mucoid degeneration, anterior cruciate ligament, acl reconstruction, arthroscopy, knee

\section{Introduction}

Mucoid degeneration, a rare degenerative condition of the connective tissue was first reported in the cardiovascular system in the early 1950s. Nevertheless, it was four decades later, in 1999, that Kumar et al reported a case of Mucoid degeneration of ACL in a 35 year old patient with posterior knee pain and restriction of terminal movements. Since then, this condition has been of particular interest to orthopaedic surgeons and speculations arouse about its diagnosis and management. Being rare and posing a diagnostic challenge, Mucoid degeneration of the ACL is one of the most misdiagnosed and misconceived condition in the field of orthopaedics. The patients are generally middle aged (average 40 years old) with a mild female predominance, presenting with insidious onset posterior knee pain for an average duration of 6 months without history of significant trauma. There is a possibility of an extension deficit with attenuated pain on terminal extension. Patient usually has an awkward antalgic gait due to limited extension in mid-stance. Anterior drawer and Lachman tests are rarely positive and the 
patient seldom complaints of instability. MRI confirms the diagnosis: 1. Abnormally thickened and ill-defined ACL; 2. Maintenance of normal orientation and continuity; 3. Increased intra-ligamentous signal (T1 and T2). 4. Possible narrowing of the intercondylar notch.

\section{Materials and Methods \\ Patients}

This is a study involving 13 patients ( 7 men and 6 women), aged between 25 to 63 years (average 41year) with varied duration of symptoms, the average being 6 months (15 days to 36 months). Approval was obtained from the Institutional Ethical Committee. Informed consent was obtained from all the patients.

\section{Clinical Manifestations}

The presenting complaint was posterior knee pain attenuating mainly on flexion (on extension for 2 patients) significantly affecting their activity of daily living such as climbing stairs, squatting or sitting cross legged. Three of them had history of fall (15 days, 4 months and 7 months prior), one of them had a twisting injury 4 months back. There was no history of significant trauma in the others. There were no signs of instability subjectively and objectively.

\section{Imaging Studies}

No significant anomaly was noted in the radiographs. MRI was taken after a failed conservative trial of NSAIDs and physiotherapy for an average duration of one month. All MRIs showed that ACL was hypertrophied and ill-defined with intraligamentous intensity. Four had an associated cystic lesion; two MRIs reported possibility of partial tear of the ACL. MRI diagnosis was made based on the following criteria:

(1) Hypertrophic ill-defined ACL,

(2) Maintenance of orientation and continuity

(3) Hyper intense intra-ligamentous signals (especially on T2 weighted images)
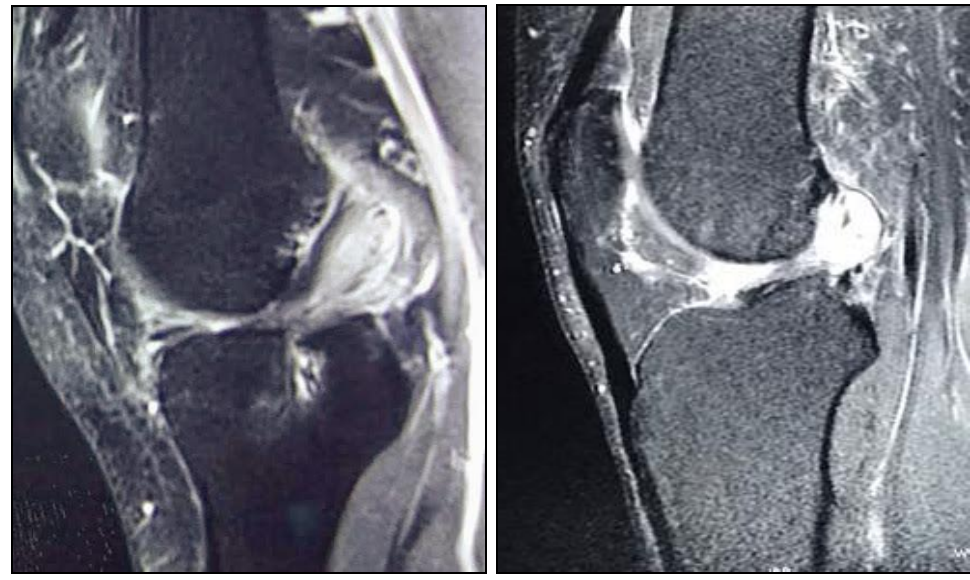

Fig 1.1 and 1.2: MRI T2 weighted image sagital view showing increased bulk of the anterior cruciate ligament suggesting Mucoid Degeneration

\section{Arthroscopic Finding}

All 13 patients underwent arthroscopy ACL reconstruction by quadrupled hamstring tendon graft. Hypertrophic ACL with yellowish degenerative materials amidst the full length of the ACL fibres were noted. Nevertheless, the ligament was found to be taut and there was no sign of any tear. Four patients had an associated Mucoid cyst at the tibial insertion site of the ACL. Seven patients had obvious intercondylar notch narrowing which constricted the ACL fibres on dynamic motion.

\section{Arthroscopic Operative Procedure}

All the surgeries were performed by a single surgeon. First, the hamstrings were harvested by a linear incision over the pes anserine region. With knee in 90 degree flexion, semitendinosis and gracilis are harvested using a tendon stripper. The graft was prepared by trimming and folding the tendons into half and securing it together with ethibond. The proximal end was looped with endobutton.

The Arthroscopic portals: Standard anterolateral and anteromedial portals were used. Diagnostic arthroscopy was performed to check the status of the meniscus, cruciate ligaments, chondral surfaces and to confirm the mucoid degeneration of ACL. The degenerated ACL was resected completely. Other pathologies such as meniscal tear and chondral damage were addressed appropriately. Narrow notch was widened by notchplasty.
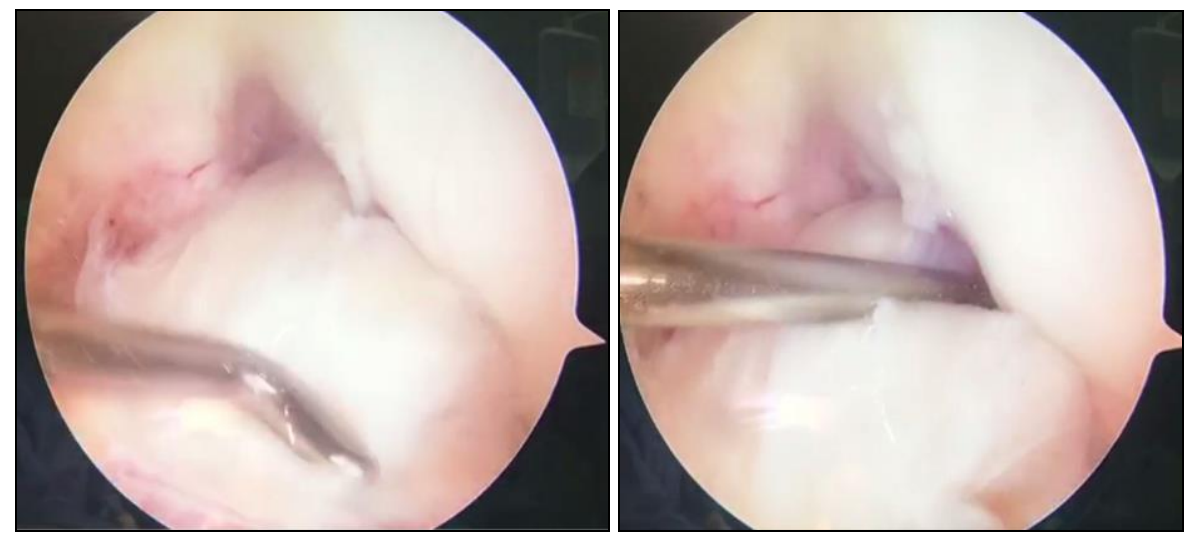

Fig 2.1, 2.2: Arthroscopic view of the Knee joint showing increased bulk of the ACL hinging on the Intercondylar notch. 


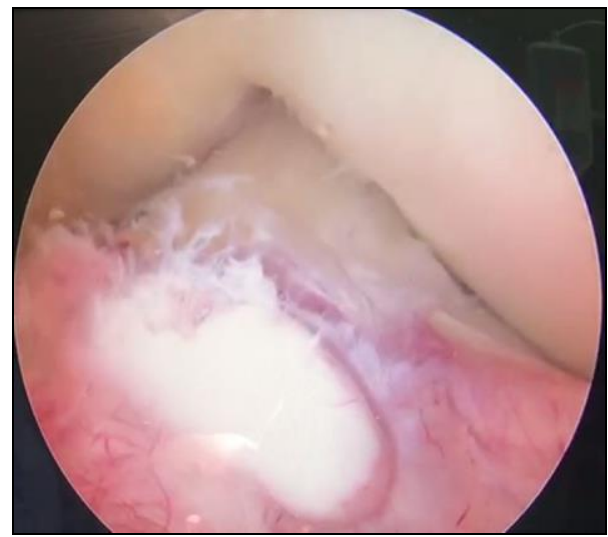

Fig 2.3: Arthroscopic view of the Knee joint showing Mucoid cyst at the tibial insertion site of the ACL
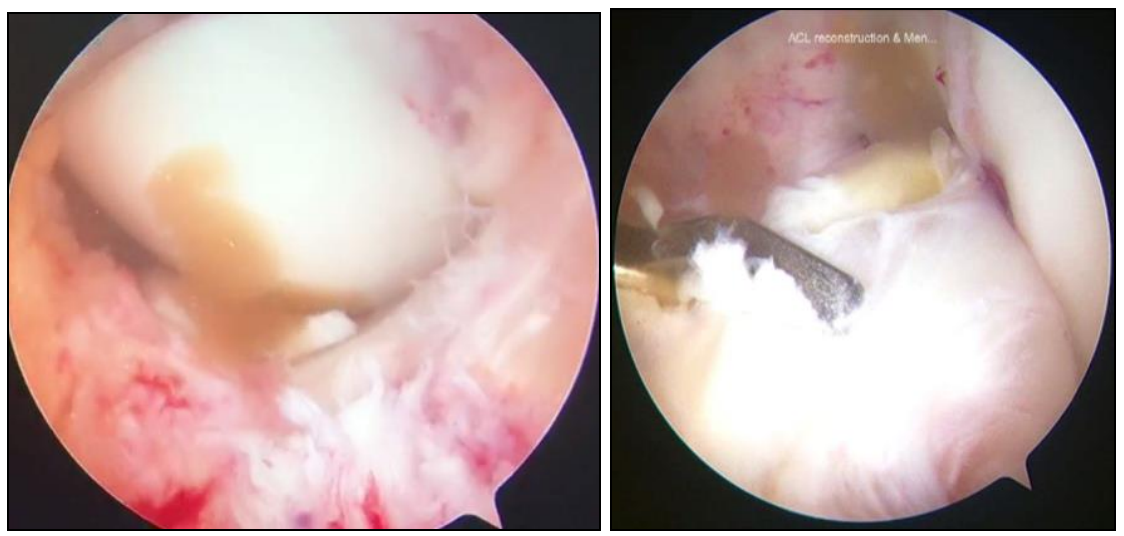

Fig 2.4, 2.5: showing the cystic fluid oozing out from the Mucoid degenerated - Anterior Cruciate ligament.

Tunnels were drilled in accordance with the width of the harvested graft. Tunneling was done on the medial wall of the lateral femoral condyle with ACL footprint as the centre point. Similarly, tibial tunnel was made with point just $5 \mathrm{~mm}$ posterior to the native ACL foot print, with tibial angle guide of 50 degrees. The graft was inserted and secured on the femoral side using the endobutton.

Cyclic loading was done by flexing and extending the knee around 20 times, which helps in positioning the endobutton over the femoral cortex without any graft loosening. Bio absorbable interference screw was used to fix the graft under tension on the tibial side. Full ROM of the knee was checked.

\section{Histopathological Studies}

Diagnosis was confirmed by histo-pathological examination by Haematoxin - Eosin stain and Alcian blue stain. Loose connective tissue with distorted collagen fibres which are healthy and uninterrupted is suggestive of mucoid degeneration. The Alcian blue stain has a high affinity for the glycosaminoglycan which is secreted by the fibroblasts in the mucoid lesion.
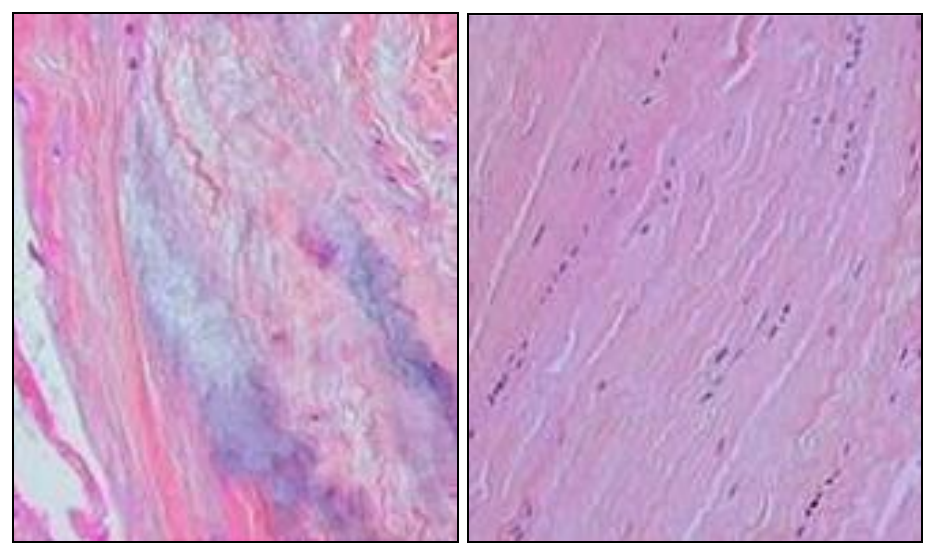

Fig 3.1, 3.2: showing Histo Pathological examination of Normal anterior cruciate ligament (left) and Mucoid degenerated ACL (right)

\section{Evaluation}

The patient was evaluated both subjectively and objectively by VAS pain score and Lysholm Knee scoring scale. Pain was quantified by the patient based on its severity on rest and terminal range of movements and scaled from 0 (no pain) to 10 (severe pain). Lysholm knee score evaluates the patient both clinically and functionally on a scale of 0 to 100 . The patients were evaluated both pre-op and at the end of followup period for an average of 15 months. A paired-t test was used to compare the outcome. Anterior drawer and Lachman tests were used at the final follow-up to assess the ligament stability. 
Arthroscopic Operative Procedure

\section{Postoperative Rehabilitation:}

Regular ACL reconstruction rehabilitation protocol was followed. Patient was initially put on knee brace and allowed to partially weight bear with support. Closed kinetic chain, hamstring and calf strengthening exercises were advised for the first 2 weeks followed by open kinetic chain and static quadriceps exercises. Passive ROM as tolerated was started from day 5 discouraging hyperextension.

\section{Results}

Out of the 13 patients, 2 patients were lost to the minimum follow-up of 1 year and hence are being exempted from the study. The remaining 11 patients were followed up for an average of 14.9 months (12 - 18 months). At the end of follow-up, all patients had a significant improvement in their knee function and activities of daily living. All but one, regained full range of motion of the knee compared to the preoperative restriction of terminal flexion (11 patients) and extension (2 patients).

There was a significant increase in the Visual Analogue Scale (VAS) pain score from an average of 5.76 to 0.54 with regard to pain in terminal range of movements. There was complete pain relief in six patients while 4 patients had a very mild discomfort on the operated knee on terminal flexion, as compared to the normal knee. The functional activity calculated by the Lysholm knee scoring scale showed an improvement from an average of 34.6 pre-op to 83.2 post-op. The anterior drawer and Lachmann tests done at the follow-up had a firm end-point and no laxity.

Table 1.1: Showing the Functional outcome of Arthroscopic ACL reconstruction for mucoid degenerated ACL evaluated by Lysholm Knee score and VAS pain Score.

\begin{tabular}{|c|c|c|c|c|c|c|c|}
\hline \multirow{2}{*}{ Patient ID } & \multirow{2}{*}{ Age } & \multirow{2}{*}{ Sex } & \multirow{2}{*}{ Follow up (months) } & \multicolumn{2}{|c|}{ Lysholm Scale } & \multicolumn{2}{c|}{ VAS } \\
\cline { 5 - 8 } & & & & Pre-op & Post-op & Pre-op & Post-op \\
\hline $\mathbf{1}$ & 46 & F & 12 & 36 & 79 & 5 & 1 \\
\hline 2 & 50 & M & 15 & 32 & 82 & 5 & 0 \\
\hline 3 & 41 & F & 12 & 39 & 80 & 6 & 1 \\
\hline 4 & 33 & M & - & 40 & - & 5 & \\
\hline 5 & 36 & & 9 & 34 & 86 & 5 & 0 \\
\hline 6 & 49 & F & 16 & 30 & 78 & 6 & 1 \\
\hline 7 & 48 & F & 9 & 28 & 82 & 7 & 0 \\
\hline 8 & 25 & M & 12 & 44 & 90 & 5 & 0 \\
\hline 9 & 63 & M & & 26 & - & 7 & \\
\hline 10 & 44 & F & 10 & 32 & 78 & 6 & 1 \\
\hline 11 & 32 & M & 18 & 3 & 87 & 7 & 0 \\
\hline 12 & 47 & F & 16 & 31 & 86 & 6 & 1 \\
\hline 13 & 26 & M & 9 & $4^{8}$ & 88 & 5 & 0 \\
\hline
\end{tabular}

One patient however, had a restriction of terminal flexion of around 15 degree (post-op) associated with mild pain (VAS 2). Nevertheless, the pain and restriction was obvious only on clinical examination and the patient was able to carry out her day-to-day activity without any hindrance. The cause of the restriction was attributed to a probability of insufficient notchplasty though there was no evidence of intercondylar notch narrowing noted intraoperatively. The MRI done for study purpose showed well uptake of the reconstructed anterior cruciate ligament with no signs of laxity or recurrence.

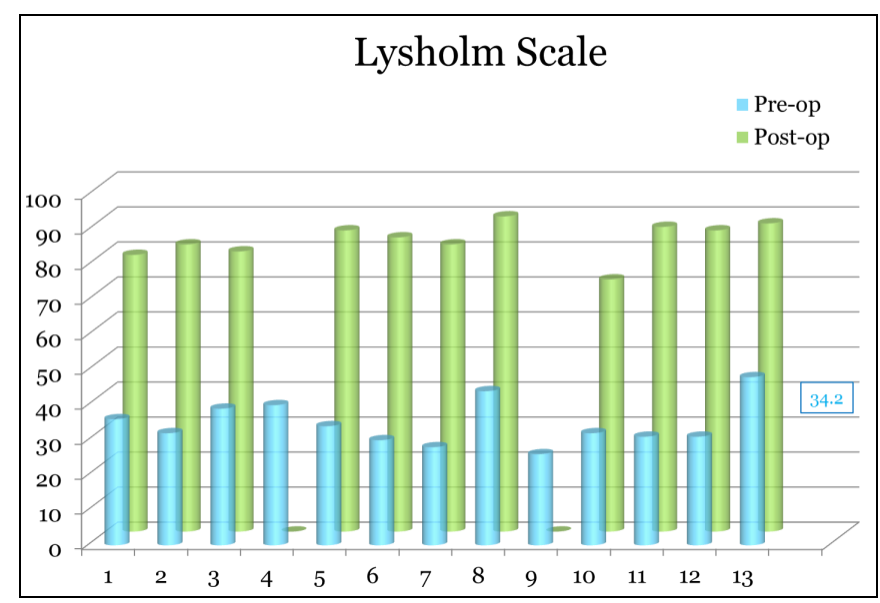

Graph 1.1: Showing the pre-op and post-op Lysholm knee scale

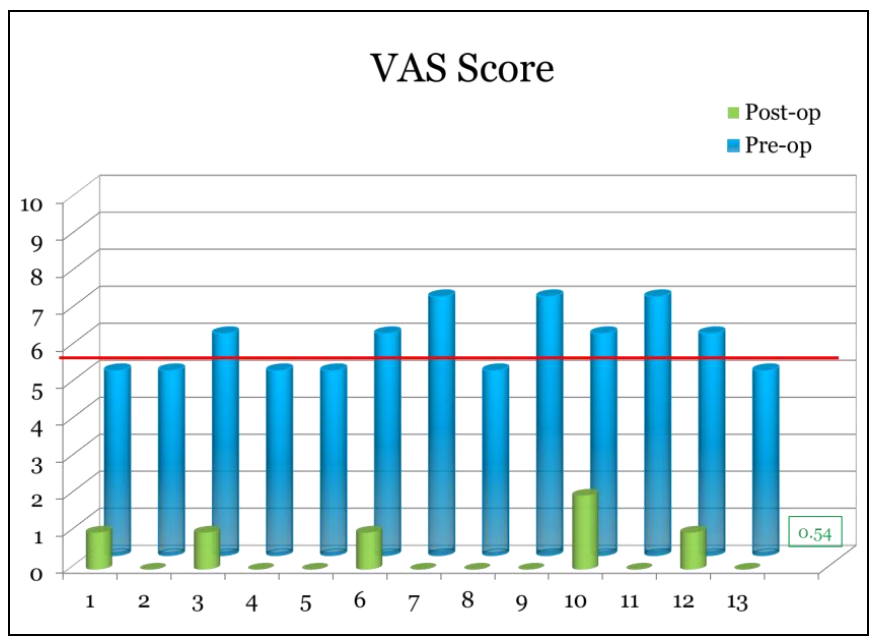

Graph 1.2: Showing the Pre-op and Post-op VAS score.

\section{Discussion}

This is the first study to assess the functional outcome of mucoid degeneration of Anterior Cruciate Ligament treated primarily by ACL reconstruction. Mucoid degeneration of the ACL is one of the most underdiagnosed and undertreated condition. Though mucoid degeneration of the ACL has been considered as a rare entity and often misdiagnosed as partial ACL tear in the MRI scan, studies have showed its prevalence as $2-5 \%$ (bergin et al, salvati et al) which are subclinical. Treatment is advised only for those patients that present clinically, with hindering of their daily activity. 


\section{Pathophysiology}

The cause of Mucoid Degeneration of ACL is not clear. Some studies have reported cases of MD of ACL following a minor trauma with insidious onset knee pain and symptoms. Other studies reported cases without any prior trauma. There are, however, many hypotheses regarding the pathophysiology of this lesion. The 'Synovial' theory states that there is invagination of the synovial lining into the substance of the ACL, causing accumulation of synovial fluid inside it. Intrinsic healing is known to be diminished in synovium deficient ACL. Cathepsin K and tartrate-resistant acid phosphatase, secreted by synovial fibroblasts have shown to cause progressive ACL degeneration in dogs. 'Degenerative' theory suggests that the formation of mucoid material is due to aging degeneration of normal tissue. This theory does not explain the formation of MD in young, active patients.

The 'traumatic' theory explains that the glycosaminoglycan formation in the mucoid degenerated ACL is due to the secretion by the fibroblasts, formed as a result of repeated micro-trauma. This theory explains the mucoid cyst and narrow intercondylar notch associated with this condition and has been supported by many authors. The tighter notch impinges on the ACL causing repeated micro traumas.

The clinical features of the MD develop only when the hypertrophic ACL impinges on the posterior cruciate ligament or on the intercondylar notch. Patients will usually present with a slow onset posterior knee pain which increases on flexion (when anteromedial bundle is involved or when the impingement is on PCL), or central knee pain aggravating on extension (when posterolateral bundle is involved or the impingement is due to narrow notch), or both. History of trauma is either insignificant or absent. When the impingement is on flexion, the patients have difficulty in squatting, sitting cross legged, or getting up from bed. When the impingement is on extension, the patients have difficulty in climbing stairs, or getting up from sitting position. The severity of the pain may be related to the amount of hypertrophy (and thereby the impingement) of the degenerated ACL.

\section{Significance of the study}

The current dictum of treatment is debridement of the degenerated parts of the ACL, removal of the interlaced mucoid materials followed by notchplasty of the intercondylar notch, if necessary. Though the degenerated parts of the ACL can be clearly viewed in the arthroscope, the debridement of the degenerative material in the ACL inter-substance is very difficult. Moreover, the extent of debridement can never be confirmed intra-operatively. Over-debridement causes laxity and instability whereas under-debridement may result in recurrence of MD. The presence of mucoid cyst near the tibial insertion site further decreases the tensile power of the ligament.

Most of the previous studies on debridement for MD of ACL are of limited sample size and lacked a long term follow-up. Though the patients are most often relieved of pain and have improved range of movements, there have been many reports of laxity and instability. Dejour et al. reported post-operative outcome of positive anterior Lachman test in 36\% and pivot shifting in $55 \%$, of the 27 patients operated. Lintz et al did a systematic Lachman test and TELOS dynamic radiographs postoperatively and showed anterior laxity in $93 \%$ of patients in 6 years follow-up.

\section{Conclusion}

Mucoid degeneration of ACL should always be suspected in a middle age patient with unexplained knee pain aggravating on flexion or extension. Arthroscopic ACL reconstruction can be a better alternative to debridement in middle aged patients and older patients with active lifestyle. In older sedentary patients, one has to consider the longer surgical time, donor site comorbidity and requirement of longer rehabilitation periods in the reconstructive procedure. Asymptomatic patients with incidental findings can be left untreated as the chances of them developing symptoms later in life are miniscule.

\section{Acknowledgement}

The authors would like to acknowledge the significant contribution of Head of department Prof. Dr. Narayana Reddy $\mathrm{M}$ and the entire faculty of the Department of Orthopaedics, CHRI and CSSH for their expert guidance and support for this study.

\section{References}

1. Kumar A, Bickerstaff DR, Grimwood JS, Suvarna SK. Mucoid cystic degeneration of the cruciate ligament. J Bone Joint Surg Br. 1999; 81(2):304-305.

2. Lancaster TF, Kirby AB, Beall DP, Wolff JD, Wu DH. Mucoid degeneration of the anterior cruciate ligament: a case report. J Okla State Med Assoc. 2004; 97(8):326328.

3. Fealy S, Kenter K, Dines JS, Warren RF. Mucoid degeneration of the anterior cruciate ligament. Arthroscopy. 2001; 17(9):37.

4. Fernandes JL, Viana SL, Mendonca JL, Freitas FM, Bezerra AS, Lima GA et al. Mucoid degeneration of the anterior cruciate ligament: magnetic resonance imaging findings of an underdiagnosed entity. Acta Radiol. 2008; 49(1):75-79.

5. Hensen JJ, Coerkamp EG, Bloem JL, De Schepper AM. Mucoid degeneration of the anterior cruciate ligament. Jbr-Btr. 2007; 90(3):192-193.

6. Hsu CJ, Wang SC, Fong YC, Huang CY, Chiang IP, Hsu HC. Mucoid degeneration of the anterior cruciate ligament. J Chin Med Assoc. 2006; 69(9):449-452

7. Lintz F, Dejour D, Pujol N, Boisrenoult P, Beaufils P. Mucoid Degeneration of the Anterior Cruciate Ligament: Selecting the best treatment option. Orthop Trauma Surg Res. 2010; 96(4):400-406

8. Makino A, Pascual-Garrido C, Rolon A, Isola M, Muscolo DL. Mucoid degeneration of the anterior cruciate ligament: MRI, clinical, intraoperative, and histological findings. Knee Surg Sports Traumatol Arthrosc, 2010.

9. McIntyre J, Moelleken S, Tirman P. Mucoid degeneration of the anterior cruciate ligament mistaken for ligamentous tears. Skeletal Radiol. 2001; 30(6):312315 .

10. Melloni P, Valls R, Yuguero M, Saez A. Mucoid degeneration of the anterior cruciate ligament with erosion of the lateral femoral condyle. Skeletal Radiol. 2004; 33(6):359-362.

11. Motmans R, Verheyden F. Mucoid degeneration of the anterior cruciate ligament. Knee Surg Sports Traumatol Arthrosc. 2008; 17(7):737-740

12. Narvekar A, Gajjar S. Mucoid degeneration of the anterior cruciate ligament. Arthroscopy. 2004; 20(2):141146. 
International Journal of Orthopaedics Sciences

13. Nishimori M, Sumen Y, Sakaridani K. Mucoid degeneration of the anterior cruciate ligament - a report of two cases. Magn Reson Imaging. 2004; 22(9):13251328.

14. Pandey V, Suman C, Sharma S, Rao SP, Kiran Acharya KV, Sambaji C. Mucoid degeneration of the anterior cruciate ligament: Management and outcome. Indian J Orthop. 2014; 48:197-202. 